Proc. 15th Int. Conference on Defects Recognition, Imaging and Physics in Semiconductors, Warsaw, Poland 2013

\title{
Characterization of Radiation Defect Centers in Neutron Irradiated Si Using Inverse Laplace Transformation to Analysis of Photocurrent Relaxation Waveforms
}

\author{
P. KAMiŃSKi*, R. KOZŁOWsKi AND J. ŻELAZKO \\ Institute of Electronic Materials Technology, Wólczyńska 133, 01-919 Warszawa, Poland
}

\begin{abstract}
High-resolution photoinduced transient spectroscopy has been applied to investigating the effect of the $1 \mathrm{MeV}$ neutron fluence on the electronic properties of radiation defects in Czochralski grown silicon in magnetic field. A new approach to the analysis of the photocurrent relaxation waveforms as a function of time and temperature has been presented. It is based on using a two-dimensional numerical procedure with implementation of the inverse Laplace transformation for creating images of the sharp spectral fringes depicting the temperature dependences of the thermal emission rate for detected defect centers. In the material irradiated with the fluence of $3 \times 10^{14} \mathrm{~cm}^{-2}$, the dominant traps with activation energies of $75 \mathrm{meV}$ and $545 \mathrm{meV}$ are tentatively identified with an aggregate of three Si interstitials and the trivacancy, respectively. In the material irradiated with the fluence by the order of magnitude higher, the activation energies of the main traps are found to be 115, 350,505, 545, and $590 \mathrm{meV}$. These traps are tentatively attributed to an aggregate of four Si interstitials, as well as to vacancy related centers such as $\mathrm{V}_{3}(2-/-), \mathrm{V}_{2} \mathrm{O}(-/ 0), \mathrm{V}_{3}(-/ 0)$ and $\mathrm{V}_{4}(-/ 0)$, respectively.
\end{abstract}

DOI: $10.12693 /$ APhysPolA.125.976

PACS: 61.72. $-\mathrm{y}, 71.55 .-\mathrm{i}, 61.80 .-\mathrm{x}$

\section{Introduction}

Silicon detectors have been recently extensively used to track high energy particles produced in experiments performed at the CERN Large Hadron Collider (LHC). These detectors are exposed to fast hadron fluences equivalent to $\approx 10^{14} \mathrm{~cm}^{-2}$ of $1 \mathrm{MeV}$ neutrons. However, for the future tracking detectors, which will be used in the designed Super LHC, the long-term operation under hadron fluences equivalent up to $\approx 10^{17} \mathrm{~cm}^{-2}$ is envisaged. To meet this challenge, a new generation of silicon-based devices exhibiting greatly improved radiation hardness must be developed.

Further development of radiation-resistant particle detectors for high-energy physics experiments requires profound knowledge on the electrical properties of irradiation-induced point defects and their formation mechanisms that could enable the defect engineering methods to be implemented. Both theoretical models and experimental results indicate that during irradiation of silicon crystal with fast hadrons, cascades of primary native defects - vacancies and selfinterstitials - arise $[1,2]$. The local density of these defects is sufficiently high that divacancies and di-interstitials are directly created [2, 3]. Moreover, the primary defects are highly mobile at room temperature and can be captured by atoms of the main impurities, such as oxygen and carbon. Since the oxygen and carbon concentrations in $\mathrm{Si}$ crystals are usually of the order of $10^{18}$ and $10^{15} \mathrm{~cm}^{-3}$,

*corresponding author; e-mail: pawel.kaminski@itme.edu.pl respectively, the irradiation with low particle fluence $\left(\leq 10^{14} \mathrm{~cm}^{-2}\right)$ mainly produces divacancies and defects created by the interaction between a native defect and an impurity, such as the vacancy-oxygen complex, carbon interstitial or carbon-oxygen complex $[4,5]$. On the other hand, the irradiation with the high particle fluence $\left(>10^{14} \mathrm{~cm}^{-2}\right.$ ) may result in producing predominantly small clusters of selfinterstitials, such as tri-interstitials or tetra-interstitials [6, 7], as well as small clusters of vacancies (multi-vacancies), such as tri-, tetra- and penta-vacancies [2, 6].

However, the electronic properties of small clusters of vacancies and selfinterstitials produced in $\mathrm{Si}$ by irradiations with high hadron fluences are poorly known. This situation is partly due to the fact that the deep level transient capacitance spectroscopy (DLTS) is not applicable, for the irradiations result in increasing the material resistivity above $10^{4} \Omega \mathrm{cm}$. The most effective method allowing determining the defect energy levels in high-resistivity silicon after irradiation with high hadron fluences is photo-induced transient spectroscopy (PITS), also referred to as photo-induced current transient spectroscopy (PICTS) [8, 9]. In this method, the levels in the bandgap are filled with charge carriers generated by optical pulses and the information about the traps properties is extracted from the photocurrent relaxation waveforms observed when the illumination is switched off. In practice, the photocurrent transients are recorded in a wide range of temperatures $(30-320 \mathrm{~K})$ with $2 \mathrm{~K}$ steps using one temperature scan and the parameters of defect centers are usually calculated in two stages.

First, the one-dimensional spectra for various emission rate windows are created by means of the correlation procedure [8]. 
Next, the temperatures corresponding to the peaks in the correlation spectra are estimated and the temperature dependences of the thermal emission rate for detected traps are determined.

Very often, however, the peaks occurring in the correlation spectra are broad, which indicates that they arise due to thermal emission of charge carriers from the different traps with very close parameters. In such cases, the single peaks corresponding to the particular traps are resolved by best-fitting the broad peaks with the sum of Gaussian functions [8].

In this paper we demonstrate a new approach to the analysis of the photocurrent relaxation waveforms as a function of two variables: time and temperature. In order to significantly increase the resolution of the PITS technique, the relaxation waveforms are transformed into the two-dimensional spectra by means of a numerical procedure based on the inverse Laplace transform algorithm [9]. The ridgelines of the sharp folds created in this way in the $3 \mathrm{D}$ space give automatically the temperature dependences of the charge carriers' emission rate for detected traps. The new approach has been implemented for characterization of radiation defect centers produced by high neutron fluences in detector-grade silicon, grown by the Czochralski method in magnetic field.

\section{Experimental details}

The samples used in this study originated from the $n$-type silicon wafers prepared from a slightly phosphorus-doped single crystal grown in $\langle 100\rangle$ direction by the Czochralski method in magnetic field (MCZ-Si). The wafers were $300 \mu \mathrm{m}$ thick and one side of them was mirror-like polished. The resistivity of as-grown samples at $300 \mathrm{~K}$ was $\approx 1 \mathrm{k} \Omega \mathrm{cm}$. The residual concentrations of oxygen and carbon atoms were $5.5 \times 10^{17} \mathrm{~cm}^{-3}$ and $2.5 \times 10^{15} \mathrm{~cm}^{-3}$, respectively. The irradiation processes with fast neutrons with an equivalent energy of $1 \mathrm{MeV}$ were carried out in a Triga type nuclear reactor. The samples were irradiated at room temperature with two neutron fluences: $3 \times 10^{14}$ (samples A) and $3 \times 10^{15} \mathrm{~cm}^{-2}$ (samples B) and before the electrical measurements were kept at this temperature for seven days. After the irradiation with the particular fluence and annealing at room temperature, the resistivity of samples $\mathrm{A}$ and $\mathrm{B}$ at $300 \mathrm{~K}$ was $\approx 3 \times 10^{5}$ and $\approx 5 \times 10^{5} \Omega \mathrm{cm}$, respectively.

For the PITS measurements, arrays of Al ohmic contact pairs were deposited on the polished surface of the irradiated samples. The width of the gap between two co-planar contacts was $\approx 0.7 \mathrm{~mm}$. The photocurrent transients' measurements were carried out in the temperature range of $30-320 \mathrm{~K}$ with an interval of $1.5 \mathrm{~K}$. The photocurrent pulses were generated by a $5 \mathrm{~mW}$ semiconductor laser emitting pulses of photons with the energy of $1.91 \mathrm{eV}$. The photon flux, controlled by the laser bias, was $1.5 \times 10^{17} \mathrm{~cm}^{-2} \mathrm{~s}^{-1}$. The duration time of the excitation pulses, generated by excess charge carriers in the region between the two contacts, was $50 \mathrm{~ms}$ and the repetition period was $263 \mathrm{~ms}$. The voltage applied between the contacts was $20 \mathrm{~V}$. The photocurrent transients were amplified using a Keithley 428 fast current amplifier and then digitized with a 12 bit amplitude-resolution and a $1 \mu \mathrm{s}$ time-resolution. In order to improve the signal to noise ratio, the digital data were averaged taking 250 transients. For further processing, each photocurrent relaxation waveform was normalized with respect to the height of the photocurrent pulse at the time of the excitation pulse termination. The detailed description of the experimental setup dedicated to characterization of defect centers in high-resistivity semiconductors has been given elsewhere [9].

The analysis of the photocurrent relaxation waveforms was performed assuming that each photocurrent relaxation waveform at a given temperature $T_{j}$ is the sum of a number of exponential components, related to the thermal emission of charge carriers from various defect centers [9]. The time constants of these components are equal to the reciprocals of the thermal emission rate $\left(e_{\mathrm{T}}\right)$ of charge carriers. The temperature dependences of emission rate for defect centers were extracted from the photocurrent relaxation waveforms by a two-dimensional numerical procedure based on the inverse Laplace transform algorithm.

First, the Laplace spectral analysis of the photocurrent relaxation waveform at the temperature $T_{j}$ was carried out by means of the CONTIN computer program developed and made available by Provencher [10].

Next, the two-dimensional Laplace spectrum $S_{\mathrm{L}}\left(T, e_{\mathrm{T}}\right)$ was formed by putting together all the one-dimensional spectra obtained as a result of the analysis of the photocurrent relaxation waveforms recorded at $J$ different temperatures.

The images of the Laplace spectral fringes for defect centers were created by projecting the sharp folds in the spectrum $S_{\mathrm{L}}\left(T, e_{\mathrm{T}}\right)$ on the plane given by the $\left(T, e_{\mathrm{T}}\right)$ axes. In this way the Laplace spectral fringes depict the temperature dependences of emission rate and are the signatures of detected defect centers. The parameters of defect centers are extracted by approximating the fringes with the lines described with the Arrhenius equation

$$
e_{\mathrm{T}}=A T^{2} \exp \left(-E_{\mathrm{a}} / k_{\mathrm{B}} T\right),
$$

where $E_{\mathrm{a}}$ is the activation energy of electron or hole traps, $A=\gamma \sigma$ is the product of a material-dependent constant $\gamma$ and the apparent capture cross-section $\sigma$ for electron or holes and $k_{\mathrm{B}}$ is the Boltzmann constant. The values of $\gamma_{n}$ and $\gamma_{p}$ for electron and hole traps in silicon are equal to $3.52 \times 10^{21}$ and $2.64 \times 10^{21} \mathrm{~s}^{-1} \mathrm{~cm}^{-2} \mathrm{~K}^{-2}$, respectively.

\section{Results and discussion}

Figure 1a and b shows the photocurrent relaxation waveforms recorded in the temperature range of 234$251 \mathrm{~K}$ for samples $\mathrm{A}$ and $\mathrm{B}$, respectively, after switching off the illumination pulse that generated electronhole pairs. It should be noted that the amplitude of the waveforms is normalized with respect of the height of the 
photocurrent pulse at the moment of the optical pulse termination. The waveforms shown in Fig. 1 represent an example of the photocurrent relaxations occurring in a temperature window in which the recombination of excess charge carriers is controlled by the thermal emission of electrons or holes from defect levels in the bandgap. The traps parameters are determined as a result of the two-dimensional analysis of the waveforms as a function of the time and temperature. It is easily seen that a relaxation waveform related to the thermal emission of charge carriers represent a slower part, ranging from 10 to $20 \%$, of the whole photocurrent decay. Moreover, the waveforms for the sample A are faster than that of the sample B. This fact indicates that in the case of the sample $A$, the thermal emission rate is higher due to the greater contribution of more shallow traps than in the case of the sample B.
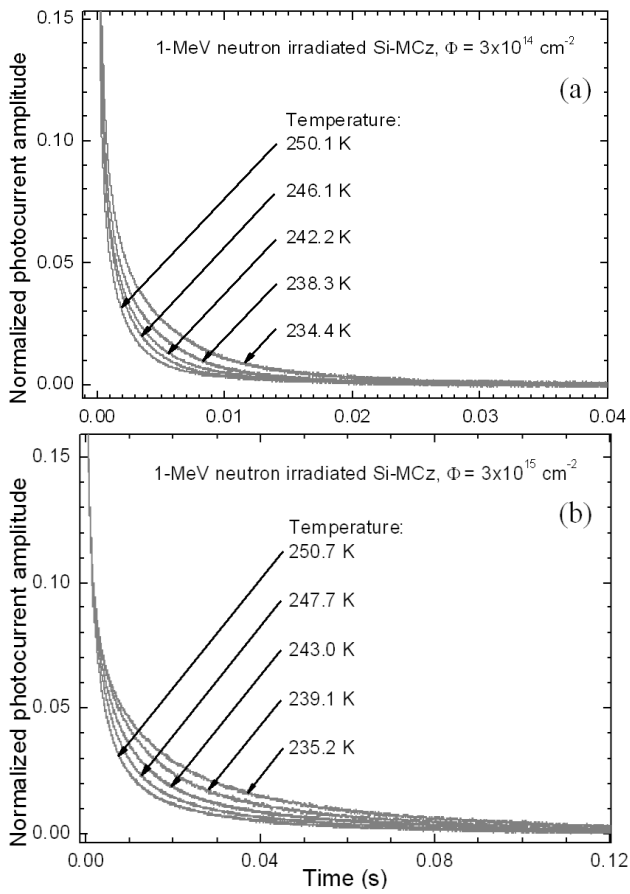

Fig. 1. Photocurrent relaxation waveforms, normalized with respect to the height of the photocurrent pulse at the moment of switching off the optical pulse, recorded in a temperature range of 234-251 $\mathrm{K}$ for MCZ-Si irradiated with $1 \mathrm{MeV}$ neutrons. (a) Neutron fluence $3 \times 10^{14} \mathrm{~cm}^{-2}$; (b) neutron fluence $3 \times 10^{15} \mathrm{~cm}^{-2}$. The relaxations are due to recombination of excess electrons and holes controlled by the thermal emission of the charge carriers from radiation defect centers.

Images of the Laplace spectral fringes for radiation defect centers detected in samples $\mathrm{A}$ and $\mathrm{B}$ are shown in Fig. 2a and b, respectively. The bars give the scale for reading the amplitude of the sharp folds on the spectral surface. It is seen that the amplitude of the fold corresponding to each trap strongly increases with rising the temperature. This fact indicates a good agreement of the experimental results with the physical model of the photocurrent relaxation. According to the model [9], the amplitude of the relaxation waveform is proportional to the emission rate of charge carriers that as shows Eq. (1) is dramatically temperature dependent. The solid lines are the signatures of detected defects and illustrate the temperature dependences of the emission rate of charge carriers. These lines were obtained directly from the approximation of the ridgelines of the sharp folds occurring on the Laplace spectral surfaces. Each solid line is described by Eq. (1) and its shape and position on the plane depends on the values of the activation energy $E_{\mathrm{a}}$ and the pre-exponential factor $A$.

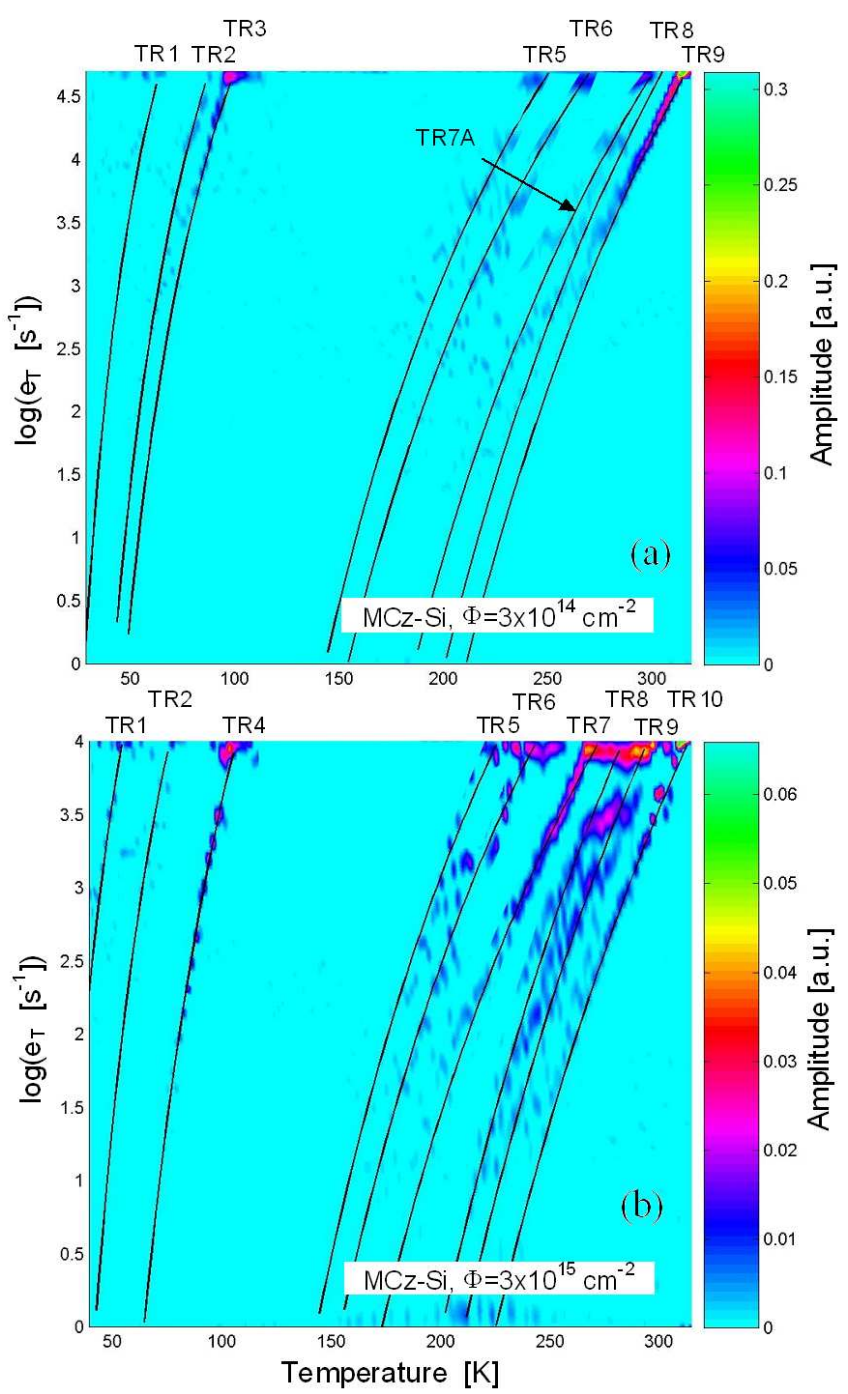

Fig. 2. Images of Laplace spectral fringes obtained by the two-dimensional analysis of the photocurrent relaxation waveforms for MCZ-Si irradiated with $1 \mathrm{MeV}$ neutrons. (a) Neutron fluence $3 \times 10^{14} \mathrm{~cm}^{-2}$; (b) neutron fluence $3 \times 10^{15} \mathrm{~cm}^{-2}$. The solid lines illustrate the temperature dependences of charge carriers' emission rate for detected defect centers.

For the material irradiated with the lower neutron fluence (sample A), eight Laplace fringes, corresponding to 
radiation defect centers labeled as TR1, TR2, TR3, TR5, TR6, TR7A, TR8, and TR9, are resolved. In the material irradiated with the higher neutron fluence (sample B), nine radiation defect centers: TR1, TR2, TR4, TR5, TR6, TR7, TR8, TR9, and TR10 are found. By comparing the results in Fig. $2 \mathrm{a}$ and $\mathrm{b}$, it is easily seen that the detected centers can be divided into three categories. Six traps: TR1, TR2, TR5, TR6, TR8, and TR9, which are present either in samples A or in samples B, belong to the first category. The second group comprises two traps: TR3 and TR7A, which are found only in samples A, irradiated with the lower neutron fluence. The third group is represented by three traps: TR4, TR7, and TR10, occurring only in samples B, irradiated with the neutron fluence by the order of magnitude higher.

The main advantage of the new approach based on the two-dimensional analysis of the temperature dependence of decay rate of the photocurrent relaxation waveforms is that it allows for visualization of all defect signatures for a broad range of emission rate values. The results shown in Fig. 2 indicate that some traps, e.g. TR1 and TR2, are observed only at higher values of the emission rate $\left(10^{3}-10^{4} \mathrm{~s}^{-1}\right)$ and other, e.g. TR8, can be well distinguished at lower values of the emission rate ranging from $10-10^{2} \mathrm{~s}^{-1}$. The temperature dependences of the emission rate for detected defect centers, which are determined on the grounds of the Laplace fringes (solid lines in Fig. 2), can be verified by comparing with the ridgelines of the folds occurring in the spectra obtained by the 2D-correlation procedure [9].

In Fig. 3a and b we illustrate the projections of the folds occurring in the correlation spectral surfaces on the plane given by the axes $\left(T, e_{\mathrm{T}}\right)$ with the superimposed solid lines being the signatures of defects detected in samples A and B from the Laplace fringes. The images shown in Figs. 3a and b confirm that the defect signatures derived from the Laplace fringes very well match the projections of the folds' ridgelines in the two-dimensional spectra obtained using the correlation procedure.

The activation energies and pre-exponential factors in the Arrhenius equation, resolved from the Laplace spectral fringes shown in Fig. $2 \mathrm{a}$ and $\mathrm{b}$, as well as tentative identifications of all radiation defect centers detected in the both kinds of samples, are summarized in Table. The results show that the activation energy of detected traps ranges from 40 to $590 \mathrm{meV}$. The shallow trap TR1 (40 meV) is likely to be attributed to a shallow thermal donor (STD) [11]. In unirradiated CZ silicon, the STDs usually are formed after heat treatment in the temperature range $300-500{ }^{\circ} \mathrm{C}$. In irradiated $\mathrm{CZ}-\mathrm{Si}$, they can be formed at room temperature due to enhanced mobility of selfinterstitials and oxygen atoms [12]. The microscopic structure of STD has not been fully understood, there are however experimental and theoretical indications that it is the complex defect composed of a silicon interstitial and oxygen atoms [13].

The activation energy of trap TR2 very well corresponds to the activation energy for hole emission from

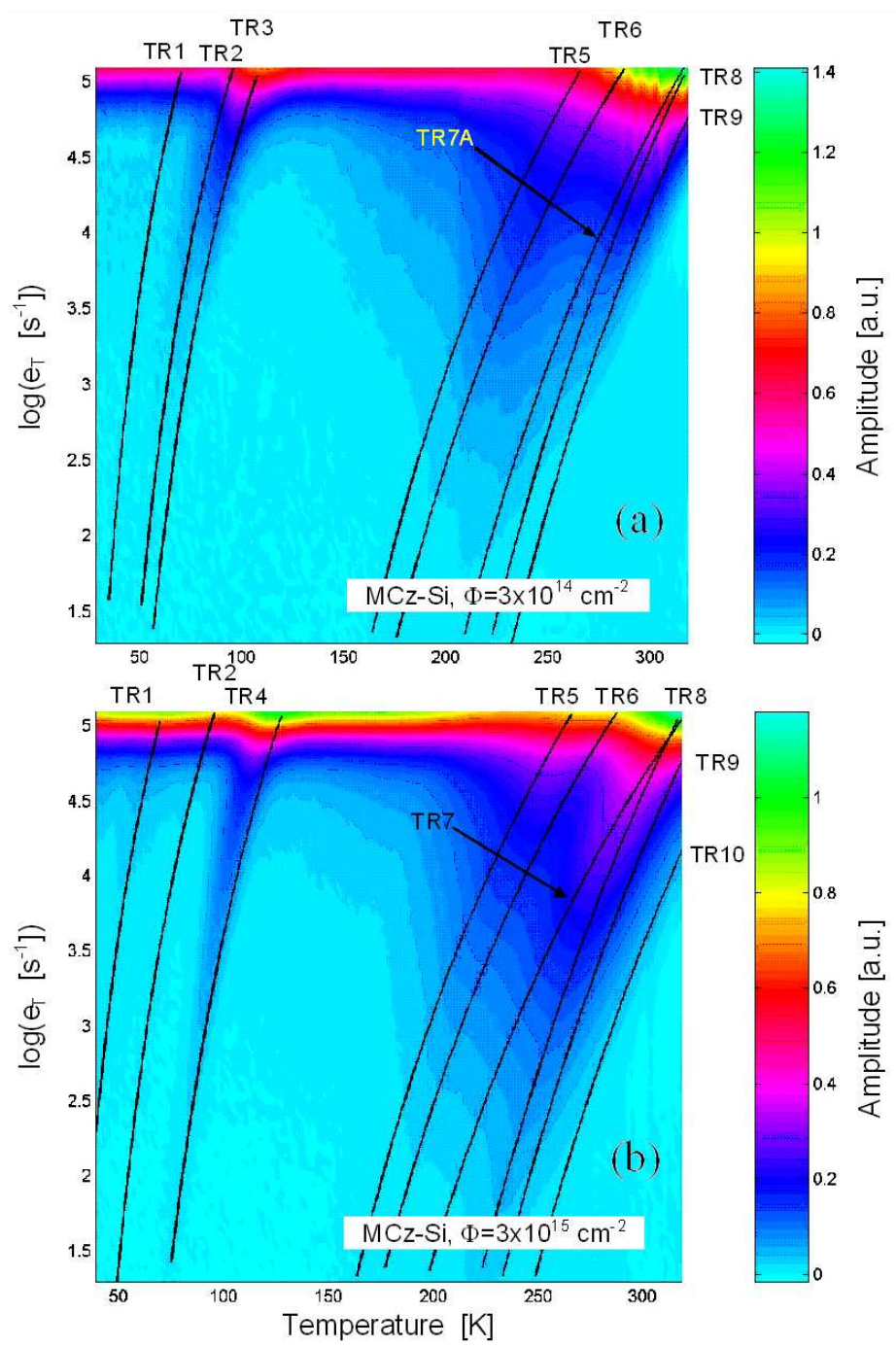

Fig. 3. Images of spectral fringes obtained from the photocurrent relaxation waveforms by the two-dimensional correlation procedure for radiation defect centers in MCZ-Si irradiated with $1 \mathrm{MeV}$ neutrons. (a) Neutron fluence $3 \times 10^{14} \mathrm{~cm}^{-2}$; (b) neutron fluence $3 \times 10^{15} \mathrm{~cm}^{-2}$. The solid lines show the temperature dependences of the emission rate of charge carriers determined from the Laplace fringes.

the interstitial carbon-substitutional carbon $\left(\mathrm{C}_{\mathrm{i}} \mathrm{C}_{\mathrm{s}}\right)$ pair in the $\mathrm{B}$ configuration [14]. This pair is a typical radiation defect in silicon, for a carbon interstitial, arising due to interaction between a silicon interstitial and carbon substitutional, can be easily captured by another carbon substitutional atom [14]. The trap TR3 is the predominant shallow trap in samples A and we have attributed this trap to the small cluster of silicon self-interstitials, namely to the tri-interstitial $\left(I_{3}\right)$. Our attributing is based on the following reasons: (i) self-interstitials are predominant point defects created directly by collision cascade and there is both theoretical and experimental evidence that they form small aggregates [3, 7, 15]; 
TABLE

Summary of parameters determined from the Laplace spectral fringes for defect centers in MCZ-Si after irradiation with neutron fluences of $3 \times 10^{14}$ (samples A) and $3 \times 10^{15} \mathrm{~cm}^{-2}$ (samples B).

\begin{tabular}{c|c|c|c|c}
\hline \hline $\begin{array}{c}\text { Trap } \\
\text { label }\end{array}$ & $E_{\mathrm{a}}[\mathrm{meV}]$ & $A\left[\mathrm{~s}^{-1} \mathrm{~K}^{-2}\right]$ & $\begin{array}{c}\text { Occurrence } \\
\text { in samples }\end{array}$ & Tentative identification \\
\hline TR1 & $40 \pm 10$ & $(1.7 \pm 0.5) \times 10^{4}$ & $\mathrm{~A}$ and $\mathrm{B}$ & $\mathrm{STD}, \mathrm{e}[11-13]$ \\
TR2 & $65 \pm 10$ & $(3.5 \pm 1.5) \times 10^{4}$ & $\mathrm{~A}$ and $\mathrm{B}$ & $\mathrm{C}_{\mathrm{i}} \mathrm{C}_{\mathrm{s}}(\mathrm{B})(+/ 0), \mathrm{h}[14]$ \\
TR3 & $75 \pm 15$ & $(2.8 \pm 0.3) \times 10^{4}$ & $\mathrm{~A}$ & $W$ line $, I_{3}, \mathrm{~h}[3,7,15]$ \\
TR4 & $115 \pm 15$ & $(2.4 \pm 0.3) \times 10^{5}$ & $\mathrm{~B}$ & $I_{4}, \mathrm{~h}[6,7,15]$ \\
TR5 & $280 \pm 15$ & $(3.4 \pm 2.5) \times 10^{5}$ & $\mathrm{~A}$ and $\mathrm{B}$ & $\mathrm{V}_{2} \mathrm{O}(2-/-), \mathrm{e}[16,17]$ \\
TR6 & $300 \pm 15$ & $(2.7 \pm 2.0) \times 10^{5}$ & $\mathrm{~A}$ and B & $\mathrm{C}_{\mathrm{i}}(+/ 0), \mathrm{h}[18]$ \\
TR7 & $350 \pm 20$ & $(2.6 \pm 1.5) \times 10^{5}$ & $\mathrm{~B}$ & $\mathrm{~V}_{3}(2-/-), \mathrm{e}[1,17]$ \\
TR7A & $415 \pm 20$ & $(4.8 \pm 1.5) \times 10^{6}$ & $\mathrm{~A}$ & $\mathrm{~V}_{2}(-/ 0), \mathrm{e}[2]$ \\
TR8 & $505 \pm 20$ & $(1.2 \pm 0.8) \times 10^{8}$ & $\mathrm{~A}$ and $\mathrm{B}$ & $\mathrm{V}_{2} \mathrm{O}(-/ 0), \mathrm{e}[16,17]$ \\
TR9 & $545 \pm 20$ & $(2.4 \pm 0.5) \times 10^{8}$ & $\mathrm{~A}$ and B & $\mathrm{V}_{3}(-/ 0), \mathrm{e}[1,17,19,20]$ \\
TR10 & $590 \pm 20$ & $(3.1 \pm 0.3) \times 10^{8}$ & $\mathrm{~B}$ & $\mathrm{~V}_{4}(-/ 0), \mathrm{e}[1,19,20]$ \\
\hline
\end{tabular}

(ii) the activation energy of trap TR3 is consistent with the activation energy for thermal quenching the photoluminescence $(\mathrm{PL}) W$ line $(1018 \mathrm{meV})$ related to exciton bound on the $I_{3}$ donor level $[3,15]$; and (iii) the calculations by density functional theory show that the $I_{3}$ donor level is placed around $E_{\mathrm{v}}+0.1 \mathrm{eV}[6,7,15]$.

The trap TR4 is the predominant shallow trap in samples B, however, it is not observed in the material irradiated with lower fluence. These facts suggest that the trap TR4 is probably formed at the expense of the trap TR3. In other words, at the higher neutron fluence, larger aggregates of silicon interstitials can be created. The possible mechanism is that at the higher density of self-interstitials, the $I_{3}$ defect captures additional silicon interstitial and the tetra-interstitial $\left(I_{4}\right)$ arises. The fact that the $I_{3}$ defect is the precursor to the $I_{4}$ defect is observed by PL measurements [15]. According to isochronal annealing experiments, the intensity of the $X$ line $(1039.8 \mathrm{meV})$ is found to increase with decreasing the $W$-line intensity [15]. On the other hand, the 1039.8 zero phonon line has been found to be related to the B3 EPR center identified with the $I_{4}$ defect $[6,7,15]$. Moreover, the calculation of the defect states show that the donor level corresponding to the bound exciton that recombines producing the $X$ line is located between 0.1 and $0.2 \mathrm{eV}$ above the valence band edge [15].

In view of the results presented in Refs. [16, 17], the traps TR5 and TR8, detected in the both kinds of samples, are attributed to the divacancy-oxygen complex in various charge states: $\mathrm{V}_{2} \mathrm{O}(2-/-)$ and $\mathrm{V}_{2} \mathrm{O}(-/ 0)$, respectively. The formation of this complex is through the interaction of migrating divacancy with interstitial oxygen [16]. The trap TR6, also found in samples A and B, is attributed to a donor center assigned to the carbon interstitial [18]. The other trap found in the both samples A and $B$ is trap TR9 assigned to the tri-vacancy $\left(V_{3}\right)$. The identification was done on the grounds of the results presented in Refs. $[1,17,19,20]$. These results show that $\mathrm{V}_{3}$ in the form of chain built up of single vacancies occurring in (110) plane gives rise to two acceptor levels located at a small distance above the midgap $[1,17,19]$. According to DLTS measurements, the two levels corresponding to $\mathrm{V}_{3}(2-/-)$ and $\mathrm{V}_{3}(-/ 0)$ are located at 0.36 and $0.46 \mathrm{eV}$ below the conduction band edge $\left(E_{\mathrm{c}}\right)$, respectively [17]. Moreover, the results of $a b$ initio calculation indicate that the first acceptor level of $\mathrm{V}_{3}$ with the $C_{2 v}$ symmetry is placed deeper in the bandgap at $E_{\mathrm{c}}-0.50 \mathrm{eV}[1,17,19]$.

The trap TR7 having the activation energy of $350 \mathrm{meV}$ is assigned to $\mathrm{V}_{3}(2-/-)[1,17]$. This trap is observed only in samples B as one of the dominant deep traps. The very deep trap TR10 is also observed only in the material irradiated with the higher neutron fluence. The latter is likely to be attributed to tetra-vacancies giving rise to the two acceptor levels $\mathrm{V}_{4}(2-/-)$ and $\mathrm{V}_{4}(-/ 0)$ located in the vicinity of the midgap $[1,19,20]$. On the other hand, the trap TR7A that is attributed to the divacancy $\mathrm{V}_{2}(-/ 0)$ is detected only in samples $\mathrm{A}$. Moreover, the intensity of the Laplace fringe corresponding to this trap is very weak, which indicates that the concentration of $\mathrm{V}_{2}$ in these samples is by an order of magnitude lower than that of $\mathrm{V}_{3}$. All these facts show that the main radiation defects produced by the neutron irradiation with the fluence of $3 \times 10^{14} \mathrm{~cm}^{-2}$ at room temperature and subsequently subjected to annealing at this temperature for the sufficiently long time are tri-vacancies and tri-selfinterstitials. The tri-vacancies can be created directly, by combining three separate vacancies $\left(\mathrm{V}+\mathrm{V}+\mathrm{V} \rightarrow \mathrm{V}_{3}\right)$, or by capturing another vacancy by the divacancy $\left(\mathrm{V}_{2}+\mathrm{V} \rightarrow \mathrm{V}_{3}\right)[1,20]$. In the material irradiated with the neutron fluence of $3 \times 10^{15} \mathrm{~cm}^{-2}$, the dominant radiation defects are tri-vacancies, tetra-vacancies and tetra-selfinterstitials. It is highly probable that the tetra-vacancies are formed by interaction of two divacancies according to the $\mathrm{V}_{2}+\mathrm{V}_{2} \rightarrow \mathrm{V}_{4}$ reaction $[1,20]$.

\section{Conclusions}

We have demonstrated a new approach to the analysis of the photocurrent relaxation waveforms making the photoinduced transient spectroscopy a high-resolution method. It is based on creating the images of defect signatures representing the temperature dependences of the emission rate of charge carriers. For this purpose, a two-dimensional analysis of the temperature-related changes in the photocurrent relaxation waveforms using the numerical procedure with implementation of the inverse Laplace transform algorithm is performed. The new approach has been exemplified by investigating the effect of fluence on the properties of electrically active defect centers induced in MCZ-Si by the $1 \mathrm{MeV}$ neutron irradiation. The results indicate that in the material irradiated with the neutron fluence of $3 \times 10^{14} \mathrm{~cm}^{-2}$ at room temperature and subjected to subsequent annealing at this temperature for the sufficiently long time, the main radiation defects are tri-vacancies and tri-selfinterstitials. In the material irradiated with the neutron fluence of $3 \times 10^{15} \mathrm{~cm}^{-2}$, the dominant radiation 
defects are tri-vacancies and tetra-vacancies, as well as tetra-selfinterstitials.

\section{Acknowledgments}

This work was carried out within the framework of CERN-RD 50 collaboration with the financial support of the Polish Ministry of Science and Higher Education under grant No. DPN/N185/CERN/2009.

\section{References}

[1] P.F. Ermolov, D.E. Karmanov, A.K. Leflat, V.M. Manankov, M, M. Merkin, E.K. Shabalina, Semiconductors 36, 1114 (2002).

[2] R.M. Fleming, C.H. Seager, D.V. Lang, P.J. Cooper, E. Bielejec, J.M. Campbell, J. Appl. Phys. 102, 043711 (2007).

[3] G. Davis, S. Hayama, L. Murin, R. Krause-Rehberg, V. Bondarenko, A. Sengupta, C. Davia, A. Karpenko, Phys. Rev. B 73, 165202 (2006).

[4] P. Kamiński, R. Kozłowski, E. NossarzewskaOrłowska, Nucl. Instrum. Methods Phys. Res. B 186, 152 (2002).

[5] I. Pintilie, E. Fretwurst, G. Lindström, Appl. Phys. Lett. 92, 024101 (2008).

[6] D. Pierreux, A. Stesmans, Phys. Rev. B 71, 115204 (2005).

[7] T. Mchedlidze, M. Suezawa, Phys. Rev. B 70, 205203 (2004).

[8] P. Kamiński, R. Kozłowski, Mater. Sci. Eng. B 9192, 398 (2002).
[9] M. Pawłowski, P. Kamiński, R. Kozłowski, S. Jankowski, M. Wierzbowski, Metrol. Measur. Syst. XII/2, 207 (2005).

[10] S.W. Provencher, Comput. Phys. Commun. 27, 213 (1982).

[11] V.V. Emtsev, G.A. Oganesyan, K. Schmalz, Appl. Phys. Lett. 68, 2375 (1996).

[12] C.P. Ewels, R. Jones, S. Öberg, J. Miro, P. Deák, Phys. Rev. Lett. 77, 865 (1996).

[13] T. Hallberg, J.L. Lindström, Appl. Phys. Lett. 68, 3458 (1996).

[14] R. Kozłowski, P. Kamiński, E. NossarzewskaOrłowska, Nucl. Instrum. Methods Phys. Res. A 476, 639 (2002).

[15] B.J. Coomer, J.P. Goss, R. Jones, S. Öberg, P.R. Briddon, J. Phys., Condens. Matter 13, L1 (2001).

[16] G. Alfieri, E.V. Monakhov, B.S. Avset, B.G. Svensson, Phys. Rev. B 68, 233202 (2003).

[17] V.P. Markevich, A.R. Peaker, S.B. Lastovskii, L.I. Murin, J. Coutinho, V.J.B. Torres, P.R. Briddon, L. Dobaczewski, E.V. Monakhov, B.G. Svensson, Phys. Rev. B 80, 235207 (2009).

[18] L. Vines, E.V. Monakhov, Yu. Kuznetsov, R. Kozłowski, P. Kaminski, B.G. Svensson, Phys. Rev. B 78, 085205 (2008).

[19] J.L. Hastings, S.K. Estreicher, P.A. Fedders, Phys. Rev. B 56, 10215 (1997).

[20] G, D. Watkins, Mater. Sci. Semicond. Proc. 3, 227 (2000). 